\title{
Managing Diversity - Gleichstellung von Frau und Mann endlich voll integriert oder nur noch Nebensache?
}

In der FAZ vom 10. März 2007 ist in einem Artikel unter dem Titel „Mustafa, Züleyha und Arminda. Immer mehr Unternehmen entdecken die Vorzüge ihrer ausländischen Mitarbeiter “ folgendes nachzulesen: „Diversity Management betreiben inzwischen viele Unternehmen in Deutschland. Hinter dem wichtig klingenden Namen versteckt sich mehr als nur die Integration ausländischer Mitarbeiter, es ist die Förderung der Vielfalt im Unternehmen allgemein“. In dem Artikel wird u.a. eine Erfolgsgeschichte bei Ford erzählt und in diesem Zusammenhang von zwei türkischen Mitarbeitern berichtet, die durch Managing Diversity höhere Aufmerksamkeit erhielten, neues Selbstbewusstsein entwickeln konnten und erweiterte Möglichkeiten im Unternehmen erhielten. An einem Beispiel wird anschaulich beschrieben, wie die Förderung und Anerkennung von kultureller Kompetenz und der Abbau von Missachtung zu Win-Win-Situationen führt, in denen erweiterte Chancen von Beschäftigten mit höheren Unternehmensgewinnen Hand in Hand gehen: „Auf einem türkischen Straßenfest in Berlin machten sie (die beiden türkischen Mitarbeiter, B.R.) Händlern das Ford-Modell Transit schmackhaft, weil sie ihnen in ihrer Muttersprache erklären konnten, wie in dem Transporter das türkische Joghurt-Getränk Ayran gekühlt werden kann“.

Im Begriff der Differenz treffen sich Managing Diversity und wissenschaftliche Diskussionen zur Kategorie Geschlecht. Ist Diversity Management als Übersetzung von Debatten über Differenzen zwischen Frauen in die Semantik von Organisationen zu interpretieren? Ist der propagierte Wandel von der monokulturellen zur multikulturellen Organisation als Weiterentwicklung von Gleichstellungspolitik zu begrüßen? Oder werden Festlegungen und Stereotypisierungen aktualisiert, die Anknüpfungspunkte für Hierarchisierungen zwischen gesellschaftlichen Gruppen bieten?

\section{Heterogenität als gesellschaftliche Ungleichheit} Seit einigen Jahren beschäftigen sich Arbeiten in der Frauen- und Geschlechterforschung mit der Frage, wie die Heterogenität zwischen Frauen aufgrund sozialer und kultureller Herkunft, sexueller Orientierungen oder des Alters in theoretischen Ansätzen und politischen Konzepten angemessen berücksichtigt werden kann. Ausgangspunkt der Debatte war die Kritik an dominanten theoretischen und politischen Konzepten, die die Lebenslagen der weißen Mittelschichtsakademikerinnen widerspiegelten. Die Diskussion war und ist mit der Suche nach verbesserten Antidiskriminierungspolitiken und veränderten Antidiskriminierungsmaßnahmen verbunden.

Der Begriff „Intersektionalität“ von der USamerikanischen Juristin Kimberlé Crenshaw beginnt sich auch im deutschsprachigen Raum zu etablieren ${ }^{1}$. Nach Crenshaw werden in amerikanischen Antidiskriminierungsgesetzen die Kategorien gender und race als sich gegenseitig ausschließend konzeptionalisiert. Insbesondere die Situation schwarzer Frauen würde in den amerikanischen Antidiskriminierungsgesetzen nicht angemessen berücksichtigt. Die Situation schwarzer Frauen vergleicht sie mit einem Unfall an einer Straßenkreuzung, an der sich die Diskriminierungsmechanismen von gender und race verschränken. Während einer weißen Frau oder einem schwarzen Mann nur auf der einen Straße etwas zustoßen kann (gender oder race), kann die schwarze Frau durch das Zusammentreffen beider Kategorien an dessen Kreuzung spezifische Verletzungen erleben ${ }^{2}$. Statt Unterdrückungsdimensionen zu addieren betont Crenshaw mit der Metapher der Straßenkreuzung die Verwobenheit und Verschränkung der Dimensionen, die sich wechselseitig verstärken, abschwächen oder auch verändern können.

Die Diskussion zur Intersektionalität erhöht die Aufmerksamkeit dafür, dass Situationen, wie die einleitend skizzierte Verkaufskommunikation, durch mehrere sich kreuzende Kategorien bestimmt sind. So wird auch eine Verkaufssituation zwischen Männern beschrieben, ohne dass dies explizit thematisiert wird. Daran schließen sich an das Managing Diversity folgende Frage an: Was ist, wenn ein Verkaufsgespräch aufgrund der Kreuzung von Kategorien wie Geschlecht, kultureller und sozialer Herkunft nicht reibungslos funktioniert? Wenn sich keine Win-Win-Situation zwischen den Zielen des Unternehmens und der Vielfalt der Mitarbeiterinnen und Mitarbeiter einstellt? Und weiter: Welche Dimensionen von Heterogenität und Vielfalt werden in genau welchen

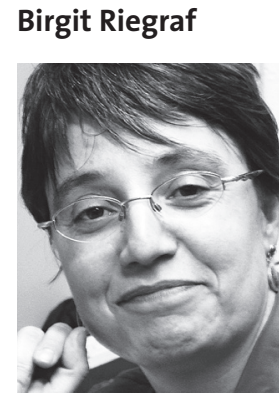

PD Dr. Birgit Riegraf Privatdozentin an der Fakultät für Soziologie, Arbeitsschwerpunkt

Frauen- und

Geschlechterforschung, Universität Bielefeld, derzeit:

Maria-Goeppert-Mayer-Gastprofessur für Internationale Frauen- und Genderforschung an der Universität Braunschweig / Institut für Sozialwissenschaft 
Situationen im Unternehmen auf welche Weise anerkannt und gefördert? Anders formuliert: Welche Dimensionen von Heterogenität und Vielfalt sind in welchen Situationen in Organisationen eher nicht erwünscht? Welche Differenzen, soziale Ungleichheiten und Abwertungen werden über organisationales Handeln erst hergestellt? Während Crenshaws Argumentation auf die gesamtgesellschaftliche Ebene abhebt, zielen diese Fragen auf Organisationen und die Wechselwirkung zwischen gesellschaftlichen und organisationalen Praxen ab.

\section{Vielfalt in Organisationen}

Die Personal- und Organisationsentwicklungsstrategie des Managing Diversity stammt von den Global Players der Privatwirtschaft, inzwischen wird aber auch in anderen Organisationen wie Schulen, NGOs und Universitäten über Managing Diversity diskutiert ${ }^{3}$. Managing Diversity ist ein in den USA entwickeltes Konzept und auch eine Reaktion auf Gesetzesvorgaben zum Schutz von Minderheiten, die zu Klagen mit hohen finanzi-

\section{Managing Diversity orientiert sich an den Zielen der jeweiligen Organisationen, was im Fall von Unterneh- men wirtschaftliche Ziele sind.}

ellen Entschädigungszahlen führen können. Sicherlich führt das Allgemeine Gleichbehandlungsgesetz in Deutschland zu einer erhöhten Aufmerksamkeitsstruktur gegenüber dem Thema. Konkrete und verbindliche Vorgaben über Diversity-Kategorien oder -Maßnahmen werden in Unternehmen jedoch nicht oder kaum gemacht. Managing Diversity orientiert sich an den Zielen der jeweiligen Organisationen, was im Fall von Unternehmen wirtschaftliche Ziele sind. Dies bringt Elisabeth Girg, Mitarbeiterin in einem Global DiversityTeam der Deutschen Bank in der FAZ vom September 2007 deutlich zum Ausdruck: „Um keine Missverständnisse aufkommen zu lassen: Diversity ist kein Sozialthema, sondern ein geschäftsorientiertes Prinzip“. Sie begründet: „Wir wollen die besten Mitarbeiter am Markt - ohne Rücksicht auf äußere Faktoren. “4 Ohne einen Bezug auf Antidiskriminierungs- und Gerechtigkeitsdebatten besteht die Gefahr, dass Vielfalt und Differenz gefördert werden, die ökonomisch opportun scheinen. Andere Differenzen können mit dem Sachzwangargument weiter ignoriert oder abgewertet werden.
Im Managing Diversity werden Differenzierungen und soziale Ungleichheiten thematisiert, als würden sie von Mitarbeiter und Mitarbeiterinnen „täglich zur Arbeit in die Organisation mitgebracht" ${ }^{5}$. Inzwischen ist aber in Untersuchungen gut belegt, dass Differenzierungen und Ungleichheiten zwischen den Geschlechtern über interaktive, symbolische und sprachliche Anordnungen, über Stellenbeschreibungen, über Arbeitszeitregelungen oder Arbeitsplatzausgestaltungen im Rahmen von Organisationen erst entstehen ${ }^{6}$. Eine umfassende Organisationsanalyse müsste sich damit beschäftigen, in welchen Kontexten, über welche Interaktionen, Praktiken und Regeln, über welche symbolische Ordnungen oder Metaphern Geschlecht, Ethnizität oder Klassen, deren Stellenwert und Relation aktualisiert und erst hervorgebracht werden.

Neu am Managing Diversity ist, dass Ungleichheiten und Differenzen von Beschäftigten betont werden, deren Bedeutsamkeit für Funktionen, Aufgaben, Zielsetzung und in den Selbstbeschreibungen von Organisationen bislang negiert wurden. Eine Reihe von Studien über Geschlecht und Organisationen standen den Neutralitätsannahmen von Organisationen seit jeher skeptisch gegenüber. „Diversity verfolgt das Ziel, Menschen mit all ihren Unterschieden zu berücksichtigen, also nicht so zu tun, als seien sie (auf irgendeiner Ebene) gleich. Aussagen wie ,Bei uns spielt es keine Rolle, ob Sie Mann oder Frau, Deutsche oder Ausländerin, hetero- oder homosexuell sind' stehen dem Diversity-Gedanken insofern entgegen, als dass es faktisch sowohl für den Einzelnen als auch für die Organisation natürlich (auf irgendeiner Ebene) einen Unterschied macht. Dies erkennt Diversity an “7. Aufgrund der Vagheit des Diversity Konzepts besteht allerdings die Gefahr, dass an gesellschaftliche Stereotype angeknüpft wird. Und: Je nach Situation können Mitarbeiter wahlweise Kategorien aktualisieren oder ausblenden, was beispielsweise in Schließungsprozessen beim beruflichen Aufstieg geschehen kann. Schon Max Weber führte aus, dass „jedes noch so äußerliche Merkmal“ wie Sprache, soziale und kulturelle Herkunft, Religion Ansatzpunkt der Ausschließung von Konkurrenten sein kann ${ }^{8}$. Folgende Schwachstellen sind in der bisherigen Praxis des Managing Diversity erkennbar:

Erstens ist die Anerkennung und Förderung von Differenzen und Ungleichheiten keineswegs immer ein harmonisches Miteinander. Dahinter können sich handfeste Interessengegensätze verbergen wie die Verteidigung von Privilegien durch dominante Gruppen. 
Zweitens lässt sich eine heterogene Gruppenzusammensetzung nicht unbedingt in ein produktives Miteinander überführen. Aufgrund differenter Interessen können unproduktive Blockaden entstehen. Nicht alle Differenzen und Ungleichheiten lassen sich einfach in betriebswirtschaftlichen Gewinn ummünzen.

Drittens besteht die Gefahr essentialistischer und stereotyper Zuschreibung, so z. B. wenn weibliche Führungskräfte als Trägerinnen „typischer weiblicher Verhaltensweisen“ eingesetzt werden ${ }^{9}$.

Viertens können Organisationen die Vielfalt von Organisationsmitgliedern nicht allein zum Ausgangspunkt von Analysen und Maßnahmen machen. Nichtanerkennungsmomente aufgrund von Alter, Ethnie oder Geschlecht werden auch über Praktiken in Organisationen erst hergestellt.

\section{Organisationslernen}

Punktuelle so genannte Diversity Trainings und eine Änderung des Unternehmensleitbildes sind gegenwärtig wohl die häufigsten Maßnahmen des Managing Diversity. In den Trainings geht es vor allem um die Sensibilisierung für Unterschiede, um Einstellungsänderungen im Sinne der Wertschätzung, dem Erlernen von Fertigkeiten, wie der Kommunikation mit Beschäftigten anderer kultureller Zugehörigkeit. Diese Maßnahmen sind nicht ausreichend, um einen umfassenden Wandel in Organisationen einzuleiten. Studien zum Organisationslernen geben Aufschluss darüber, unter welchen Bedingungen Organisationen in der Lage sind, sich einer grundlegenden Selbstreflexion z.B. im Umgang mit der Kategorie Geschlecht zu unterziehen und Innovationen im Geschlechterverhältnis anzustoßen. Aus dieser Perspektive müssen zwei Voraussetzungen erfüllt sein: zum einen sind das ist aus Gleichstellungsstrategien in Organisationen bekannt - Strategien auf eine längerfristige Perspektive auszurichten, damit sie erfolgreich sein können. Zum anderen müssen permanente und längerfristige Zweifel über bereits erreichte Fortschritte institutionalisiert werden, um Widersprüchlichkeiten, Ambivalenzen und Mehrdeutigkeiten (z. B. festzulegen, was aufgelöst werden soll, wie im Beispiel der weiblichen Führungskraft) von Erfolgen immer wieder neu diskutieren und reflektieren zu können. Mit anderen Worten: Erfolgreiche Lernprozesse im Bezug auf die Geschlechterverhältnisse - so eine Erkenntnis aus der Organisationssoziologie - sind nur über die Institutionalisierung längerfristiger, immer wieder selbstreflexiver Prozesse möglich - zum Beispiel in Form von prozessevaluierender Expertisen oder längerfristiger Beratungstätigkeiten. Punktuelle Maßnahmen führen nicht zu längerfristigen Innovationen, sondern das Spannungsverhältnis zwischen Routine und Innovation wird dann zugunsten der Routine aufgelöst ${ }^{10}$.

Grundlegende Umstrukturierungsprozesse in Organisationen werden durch externe „Schocks“ erleichtert, wenn also Organisationen in eine existenzielle Krise geraten. In solchen Situationen kann es zu einem Wechsel in den Führungspositionen, der Anerkennung von Organisationsdissidenten oder zu hoher Experimentierbereitschaft kommen, wodurch beispielsweise Gleichstellungsmaßnahmen eine höhere Aufmerksamkeit erhalten können ${ }^{11}$.

\section{Literatur:}

Allmendinger, Jutta/Podsiadlowski, Astrid (2001): Segregation in Organisationen und Arbeitsgruppen, in: Heintz, Bettina (Hg): Geschlechtersoziologie, Kölner Zeitschrift für Soziologie und Sozialpsychologie. Sonderheft Nr. 41, Opladen, 276-307

Burdas, Corinna (2007): Mustafa, Züleyha und Arminda. Immer mehr Unternehmen entdecken die Vorzüge ihrer ausländischen Mitarbeiter, in: Frankfurter Allgemeine Zeitung, 10. März

Crenshaw, Kimberle Williams (2000): The Intersectionality of Gender and Race Discrimination, ttp://www.womensplace.osu.edu/Archives/crenshaw.pdf

Frohnen, Anja (2005): Diversity-in-Action. Multinationalität in globalen Unternehmen am Beispiel Ford, Bielefeld

Hansen, Katrin/Müller,Ursula (2003): Diversity in Arbeits- und Bildungsorganisationen, in: Belinszki, Eszter/ Hansen, Katrin/ Müller, Ursula $(\mathrm{Hg})$ : Diversity Management. Best Practices im internationalen Feld, Münster, 9-60

Heintz, Bettina/Nadai, Eva/Fischer, Regula/Ummel, Hannes (1997): Ungleich unter Gleichen, Frankfurt a. M./ New York

Knapp, Gudrun-Axeli (2005): ,Intersectionality“ - ein neues Paradigma feministischer Theorie? Zur transatlantischen Reise von „Race, Class, Gender", in: Feministische Studien 23, 68-81

Koall, Iris (2001): Managing Gender und Diversity. Von der Homogenität zur Heterogenität in der Organisation der Unternehmung, Münster

Krell, Gertraude (2004): Managing Diversity: Chancengleichheit als Wettbewerbsfaktor, in: Krell, Gertraude (Hg): Chancengleichheit durch Personalmanagement. 4.Aufl., Wiesbaden, 41-56

Obermeier, Birgit (2007): Es lebe der Unterschied, Frankfurter Allgemeine Zeitung, FAZ-Net vom 17. November

Rastetter, Daniela (2007): Managing Diversity: Eine für die Gleichstellung von Frauen gewinnbringende Strategie?, in: aktuelle informationen 2007 Heft 2, 23f.

Riegraf, Birgit (1995): Mikropolitik und Geschlecht, Opladen

Riegraf, Birgit (2000): Organisationswandel, Organisationslernen und das Geschlechterverhältnis, in: Lenz, Ilse/Nickel, Hildegard Maria/ Riegraf, Birgit (Hg): Geschlecht, Arbeit, Zukunft, Münster, 150-177

Riegraf, Birgit/Zimmermann, Karin (2005): Der Wandel der Wissensordnung in der Wissensgesellschaft und die Kategorie Geschlecht. Annäherungen an ein Forschungsdesiderat, in: Funder, Maria/Dörhöfer, Steffen/Rauch, Christian (Hg): Jenseits der Geschlechterdifferenz? Geschlechterverhältnisse in der Informations- und Wissensgesellschaft, München und Mering, 21-37

Stuber, Michael (2004): Das Potenzial von Vielfalt nutzen - Den Erfolg durch Offenheit steigern, Neuwied/Kriftel

Walgenbach, Katharina/Grohs, Telse S. (2006): Interdependenzen-Klassen-Geschlecht-Ethnie, http://www2.gender.hu-berlin.de/geschlecht-ethnizitaet-Klasse

Wilz, Sylvia M. (2007): De-Institutionalisierung, Individualisierung und Personalisierung?, in: Aulenbacher, Brigitte/Funder, Maria/Jacobsen, Heike (Hg): Arbeit und Geschlecht in der modernen Gesellschaft. Forschung im Dialog, 114-130

\footnotetext{
1 Z.B. Knapp 2005.

2 Walgenbach/Grohs 2007; Crenshaw 2000.

3 Koall 2001; Hansen/Müller 2003; Krell 2004; Rastetter 2007.

4 Obermeier 2007.

5 Frohnen 2005

6 Riegraf 1995; Heintz et al. 1997; Allmendinger/Podsiadlowski 2001.

7 Stuber 2004: 20.

8 Vgl. hierzu Wilz 2007

9 Vgl. Krell 2004.

10 Riegraf 2000; Riegraf/Zimmermann 2005.

11 Riegraf 2000.
} 